\title{
Intense Ion Beam Propagation in a Reactor Sized Chamber
}

\author{
J.-L. Vay \\ LBNL, Berkeley, CA, USA \\ C. Deutsch \\ LPGP, Université Paris XI, 91405 ORSAY, France
}

August 28, 2000

\begin{abstract}
We consider the physics of the ballistic transport of intense ion beams in a heavy ion fusion reactor chamber filled with low pressure FLIBE gas. We consider first a single beam envelope model and show via a simple case that emittance growth is an issue in the chamber as well as in the accelerator. We develop a model for the neutralization of beam spacecharge by the electrons produced by gas ionization by the beam and derive an expression for the evolution of the neutralization factor as the beam propagates into the chamber. We then extend the envelope model from a one species beam to a beam of ions of several charge states by considering the entire beam as a set of subbeams (one for each charge state) each described with coupled envelope equation. The fully electromagnetic PIC code BPIC was used to investigate the behavior in greater detail. A parametric study of the sensitivity of the final spot radius at the target versus the ion beam stripping and gas ionization cross-sections (which are characterized by large uncertainties) shows that, in the studied regime (Hylife-II parameters), the accessible window of cross-sections for ballistic transport in the chamber through neutral FLIBE gas is eventually small. The temperature evolution for each species and the emittance growth for the entire ion beam was studied for a typical scenario and indicates that a fair amount of the initial electric potential energy carried by the beam
\end{abstract}


as it enters the chamber is converted into temperature and transverse emittance. The high temperature of the ionization-produced electrons prevents a full charge neutralization of the ion beam as it approaches the target. It is shown that focusing a beam array or pre-ionizing a fraction of the background gas may help in reducing the focal spot.

Keywords: Heavy Ion Fusion, Intense Ion Beams, Reaction Chamber.

P.A.C.S. Numbers: $28.50 \mathrm{Re}, 41.75 \mathrm{Ak}, 41.80 \mathrm{Gg}, 29.27 \mathrm{Bd}$

\section{Introduction}

We investigate the beam ballistic transport in a HIF fusion chamber using three levels of description: single envelope model, multi-envelope model and ParticleIn-Cell (PIC) model. While the PIC model gives a detailed and accurate description, the envelope models provide simplified 'ideal' cases to which the PIC model results can be compared, highlighting the importance of various effects. Especially, the importance of emittance growth during chamber transport is given here a specific attention. The envelope models are 'slice' models by nature while the PIC model can describe the entire space. The experience acquired with the envelope models was used to develop a slice PIC description. Such a description may especially be useful for beam array chamber transport simulation and an example is given.

\section{Modeling tools}

\section{- Single envelope model: BENV}

Neglecting the details of every particle trajectory in the ion beam, we use the envelope equation 


$$
\frac{d^{2} R}{d z^{2}}=\frac{K}{R}+\frac{\varepsilon^{2}}{R^{3}}, \quad K=\frac{Z e I}{2 \pi \varepsilon_{0} m_{0} c^{3} \beta^{3} \gamma^{3}}(1-f)
$$

where $\mathrm{R}$ is the outer beam radius, $\varepsilon$ is the beam emittance, $\mathrm{z}$ is the beam center location in the laboratory frame, $\mathrm{K}$ is the generalized perveance where we have assumed a comoving neutralizing electron beam through the neutralizing factor $\mathrm{f}, \mathrm{Z}$ is the beam ion mean charge (averaged over all possible charge states), I is the ion beam current and $\mathrm{m}_{0}$ is the beam ion rest mass.

\section{- Neutralization factor model}

When the ion beam flows through the background gas, the latter experiences ionization of part of its initial molecules. The resulting positive charges leave the beam trajectory (in our case longitudinally) and the negative ones get trapped by it until charge neutralization is complete. In [1] (Eq. (4.293), p.279), the neutralization factor is given by $f=1-\exp \left(-n_{g} \sigma_{i o} \beta c t\right), t \geq 0$ where $\mathrm{t}$ is the time, $\mathrm{n}_{g}$ is the background gas density and $\sigma_{i o}$ is the background gas ionization (from collision with a beam ion) cross-section. This expression was established assuming that all the neutralizing electrons are available for neutralization as soon as their are created by ionization of the background gas molecules. This is not true in our case where an electron is available for neutralization only when the corresponding ion of $\mathrm{BeF}_{2}^{+}$or $\mathrm{LiF}^{+}$created from gas molecule ionizations has left the beam longitudinally (the beam space-charge is too weak to expel them radially in the time of consideration). This leads to a retardation term $\tau$ in the formula which is then given by $f=1-\exp \left(-n_{g} \sigma_{i o} \beta c[t-\tau]\right), t \geq 0$ with $\tau=\left(1-\exp \left[-\beta c t / l_{b}\right]\right) l_{b} / \beta c$ where $l_{b}$ is the beam length. 


\section{- Multi-envelope model: BMENV}

In order to get a direct physical insight into the charge state dependence of ion beam focusing, we consider a separate envelope equation for every ion beam charge state (at which is associated a comoving neutralizing electron beam). Thus, the entire ion beam appears as a superposition of several coaxial sub-

beams. The population $\mathrm{N}_{i}$ of the $\mathrm{i}^{\text {th }}$ beam (charge state $\mathrm{i}$ ) evolves in time according to

$$
\frac{d N_{i}}{d t}=\left[N_{i-1} \sigma_{S t}(i-1 \rightarrow i)-N_{i} \sigma_{S t}(i \rightarrow i+1)\right] n_{g} \beta c
$$

in terms of Flibe gas density $\mathrm{n}_{g}$, relative beam-gas velocity $\beta c$ and cross-sections $\sigma_{s t}(\mathrm{i} \rightarrow \mathrm{i}+1)$ which account for the stripping of charge state $\mathrm{i}$ ions through collisions with the residual gas. Then, a given beam of charge state i may be given the envelope equation (given here in the beam frame)

$$
\frac{d^{2} R_{i}}{d t^{2}}=\frac{\kappa_{i}}{R_{i}}+\frac{\beta^{2} c^{2} \varepsilon^{2}}{R_{i}^{3}}
$$

with

$$
\kappa_{i}=\frac{i e^{2} \sum_{j=1}^{n}\left[N_{j}\left(\begin{array}{cc}
R_{i}^{2} / R_{j}^{2} & , R_{i}<R_{j} \\
1 & , R_{i} \geq R_{j}
\end{array}\right)-N_{e j}\left(\begin{array}{cc}
R_{i}^{2} / R_{e j}^{2} & , R_{i}<R_{e j} \\
1 & , R_{i} \geq R_{e j}
\end{array}\right)\right]}{2 \pi \varepsilon_{0} l_{b} m_{0}}
$$

sum of all electrostatic fields acting upon subbeam i. For each $\mathrm{j}$ there is an associated beam of ions of charge state $\mathrm{j}$ plus the electrons produced from beam stripping (first term in the squared bracket). Because these electrons are expected either to stay with the beam or to be replaced by electrons issued from 
gas ionization, they are taken into account directly into the first term where we consider the total charge to be (je). $\mathrm{N}_{j}$ (contribution from the ions stripped to the $+\mathrm{j}$ charge state $)+(\mathrm{j}-1) \cdot(-\mathrm{e}) \cdot \mathrm{N}_{j}$ (contribution from the electrons resulting from these stripping) $=\mathrm{e} . \mathrm{N}_{j}$. Additionally, a subbeam of radius $\mathrm{R}_{e j}$ transporting $\mathrm{N}_{e j}$ neutralizing electrons (second term in the squared bracket) produced from the background gas ionization accompanies each ion subbeam j. Every subbeam is given the same length $l_{b}$. For each $\mathrm{j}$, the t-dependent neutralization factor reads as $f_{j}(t)=1-\exp \left(-n_{g} \sigma_{i o}(j) \beta c[t-\tau]\right)$ with $\tau=\left(1-\exp \left[-\beta c t / l_{b}\right]\right) l_{b} / \beta c$ and the corresponding number of neutralizing electrons $\mathrm{N}_{e j}$ for each subbeam $\mathrm{j}$ is then given by $N_{e j}(t)=N_{j}(t) \cdot f_{j}(t)$ with $\sigma_{i o}(\mathrm{i})$, Flibe gas ionization cross section by beam ions with charge state $\mathrm{i}$. We take $\mathrm{R}_{e j}=\max \left(R_{j}, \mathrm{R}_{e \min }\right)$ where $\mathrm{R}_{e \min }$ is an arbitrary minimum radius accounting for the high mobility of electrons near minimum focusing.

\section{- Particle-In-Cell modeling: BPIC}

A full self-consistent and detailed description of the problem calls for a Particle-In-Cell modeling and we have used for that purpose the particle code BPIC [2]. BPIC is an electromagnetic Particle-In-Cell code using the MonteCarlo-Collision algorithm to model the gas ionization by the beam ions and the beam stripping. It allows to perform the calculations either in 3D (Cartesian XYZ), 2D (Cartesian XY, axisymmetric RZ) or 1D (Cartesian X, axisymmetric $\mathrm{R}$ ). In this paper, we will present calculations performed with the RZ, XY and $\mathrm{R}$ geometries. In the $\mathrm{XY}$ and $\mathrm{R}$ geometries, only a slice of the beam is modeled. In these slice modes, the treatment of beam neutralization requires a time-dependant estimation of the number of neutralizing electrons created 
in the simulated area at each time step. For this, we take the model developed previously for the neutralization factor as applied in BMENV giving the number of neutralizing electrons summed over all the beam ion charge states $N_{e}(t)=\sum_{j} N_{e j}(t)=\sum_{j} N_{j}(t) \cdot f_{j}(t)$. The position of each new neutralizing electron created at each time step is randomly created inside the ion beam. BPIC calculations in slice mode ( $\mathrm{R}$ or $\mathrm{XY}$ geometry) offers a reduced model compared to calculations in RZ (or XYZ) geometry, yet more detailed and selfconsistent than with the BMENV model. The interest of the XY mode is to allow beam array modeling at a lower computational cost than a full threedimensional calculation.

\section{Results}

In all the calculations, we have used the parameters of the Hylife-II power plant $\left(\mathrm{Z}=1, \mathrm{I}=4688 \mathrm{~A}, \mathrm{~m}_{0}=210 \mathrm{amu}, \beta=0.3\right)$, assumed $\varepsilon \approx 11 \pi \mathrm{mm} . \mathrm{mrad}$, an initial radius $\mathrm{R}_{0}=5.2 \mathrm{~cm}$ and a focusing length $\mathrm{L}=\mathrm{R}_{0} /(\mathrm{dR} / \mathrm{dZ})_{0}=2.6 \mathrm{~m}$.

\section{- using the single envelope code BENV}

To better understand the effect of Flibe for chamber transport, we first compute the focal spot radius assuming no Flibe. Using BENV, we computed the minimum radius of focusing $\mathrm{R}_{\text {min }}$ due to: perveance only $(\varepsilon=0) \Rightarrow \mathrm{R}_{\min } \approx$ $0.7 \mathrm{~mm}$, emittance only $(\mathrm{f}=1) \Rightarrow \mathrm{R}_{\min } \approx 1.8 \mathrm{~mm}$, both effects $(\varepsilon \neq 0, \mathrm{f}=0) \Rightarrow \mathrm{R}_{\min }$ $\approx 3.1 \mathrm{~mm}$. We observe that most of the minimum radius is due to the emittance. What happen if the beam emittance is not preserved in the chamber? If we assume a linear progression of the emittance from $\varepsilon \approx 11 \pi \mathrm{mm}$.mrad at $\mathrm{z}=0$ to $\varepsilon \approx 22 \pi \mathrm{mm}$.mrad at $\mathrm{z}=\mathrm{L}$ and then a stabilization onto a plateau, we find $\mathrm{R}_{\min }$ 
due to: emittance only $(\mathrm{f}=1) \Rightarrow \mathrm{R}_{\min } \approx 3.4 \mathrm{~mm}$, both effects $(\varepsilon \neq 0, \mathrm{f}=0) \Rightarrow \mathrm{R}_{\text {min }}$ $\approx 5.1 \mathrm{~mm}$. This shows that the emittance has also to be preserved in the chamber as much as possible, even assuming complete neutralization of the beam spacecharge.

\section{- parametric study varying cross-sections}

Because the considered cross-sections values are still controversial, we made a parametric study of the focal spot radius versus the beam stripping and gas ionization cross-sections. We considered five series of stripping cross-sections $\sigma$ (beam stripping) $\equiv \sigma_{s t}=\mathrm{n}_{s t} \sigma_{s t}^{*} \cdot 10^{-20} \mathrm{~m}^{2}$ with $\mathrm{n}_{s t}=0,1,2,3,4$ and six series of gas ionization cross-sections $\sigma$ (gas ionization) $\equiv \sigma_{i o}=\mathrm{n}_{i o} \sigma_{i o}^{*} \cdot 10^{-20}$ with $\mathrm{n}_{i o}=0$, $1,2,3,4,5$, where $\sigma_{s t}^{*}$ and $\sigma_{i o}^{*}$ are given varying with the projectile charge state by $\sigma_{s t}^{*}=(1 ., 0.665,0.335,0.12,0.06,0.03,0.015,0.007,0.0035,0.0018)$ and $\sigma_{i o}^{*}=(1 ., 1.22,1.49,1.82,2.21,2.65,3.15,3.71,4.33,5.00)$. More information on these cross-sections are given in [3].

Using the multi-envelope code BMENV with $\mathrm{R}_{e \min }=0$ and a constant emittance $\varepsilon=11 \pi \mathrm{mm}$.mrad, we obtained the focal spot radius containing $95 \%$ of the particles in function of the gas ionization and beam stripping cross-sections plotted on Fig.1.a).

The same exercise (focal spot radius versus cross-sections) has been done with the particle code BPIC in RZ mode. For simplicity, the Flibe was reduced in the simulations to its main component $\mathrm{BeF}_{2}$ which constitutes $90 \%$ of it (the $10 \%$ of $\mathrm{LiF}$ was replaced by $\mathrm{BeF}_{2}$ ). The results are displayed on Fig.1.b). We observe that BMENV predicts a smaller focal spot than BPIC for $\sigma$ (gas ionization) larger than $0.6^{*} 10^{-16} \mathrm{~cm}^{2}$. By default, BMENV assumes 
a constant emittance and assumes that the neutralizing electron subbeam radii equal the ion subbeam radii. As it will be shown in the RMS data results, the emittance does not stay constant during chamber propagation. Also, for $\mathrm{n}_{s t}=4$ and $\mathrm{n}_{i o}=5$, we plotted on Fig.1.c) a snapshot of the $95 \%$ envelopes for the ion beam, the electron population and the Flibe ions when about one third of the ion beam has passed beyond the target position. While the gas ions lag behind the beam, confirming that they escape the beam longitudinally, it is clear that the electrons are very mobile and expand far away from the ion beam.

This motivated us to make another series of runs with BMENV considering $\mathrm{R}_{e \min }=1 . \mathrm{cm}$ and $\varepsilon=11 \pi \mathrm{mm}$.mrad for $\mathrm{t}<7 \mathrm{~ns}$ and then a ramp going from $11 \pi \mathrm{mm} . \mathrm{mrad}$ to $\left(1+1.5^{*}\left(\mathrm{n}_{s t^{-}}\right) / 5\right)^{*} 11 \pi \mathrm{mm} \cdot \mathrm{mrad}$ between $\mathrm{t}=7 \mathrm{~ns}$ to $\mathrm{t}=30 \mathrm{~ns}(\mathrm{the}$ choice of the ramp seems arbitrary but will become clear from the RMS data BPIC results). The result is displayed on Fig.1.d). We note that the obtained results are then very close to the ones obtained with BPIC.

These results show that for a given value of the beam stripping cross-section, there is a plateau as the gas ionization cross-section increases. This means that, although more neutralizing electrons are provided for high values of gas ionization cross-section, electrons temperature and ion beam emittance growth seem to prevent the beam from converging to a smaller focal spot.

\section{- RMS data}

The RMS data provide additional details. On Fig. 2.a)b)c), we display the time history of the beam RMS radius (entire beam at fixed time), the beam emittance (average of beam slices at fixed time) and the beam ions temperature (average of beam cells at fixed time) for the $\mathrm{n}_{s t}=4$ and $\mathrm{n}_{i o}=5$ case (labeled as 
'Flibe') and also in the case of vacuum transport with perveance (case 'vacuum') or not (case 'vacuum, no perveance'). We observe that the propagation through Flibe produces a fair amount of emittance growth with temperature gain compared to the other cases, resulting in a larger minimum RMS radius. The background gas electrons temperature (average of population cells at fixed time) history is displayed on Fig.2.d). There is a first rise to a plateau attributed to acceleration into the electrostatic beam field followed by a peak coinciding with the beam ion temperature one. This electron temperature rise explains the picture described previously of electrons traveling somewhat far away of the beam and neutralizing only partially its space-charge.

These results can be understood if we remark that the ion beam carries at the chamber entrance a formidable amount of electrostatic energy of about $68.10^{6} \mathrm{TeV}$, corresponding to an average of about $433 \mathrm{keV}$ per beam ion. If the beam space-charge gets to be neutralized, this energy becomes free energy. The simulations indicate that part of it is transformed into beam ion temperature, ion beam deformation (these two effects causing beam emittance growth) and electron temperature (this effect resulting in incomplete beam space-charge neutralization).

\section{- Percentage of particles in a given radius}

Still using $\mathrm{n}_{s t}=4$ and $\mathrm{n}_{i o}=5$, we modeled chamber transport of an initial $5.2 \mathrm{~cm}$ radius axisymmetric beam, with BPIC in modes $\mathrm{RZ}, \mathrm{R}$ and XY. On Fig.3, we display the minimum radius in which a given percentage of the beam passed through. The results are shown in the case of perveance turned off $(\mathrm{f}=1)$ or on (others). We observe that the results are very close to each other 
whatever the chosen geometrical mode of calculation, giving some confidence in the slice mode model and cross-checking the electromagnetic solvers, being different between the axisymmetric $\mathrm{R}$ mode and the Cartesian XY mode. In the $\mathrm{XY}$ mode, we are not restricted to axisymmetric beams and we have also performed a calculation where the $5.2 \mathrm{~cm}$ radius axisymmetric beam has been divided into four $2.6 \mathrm{~cm}$ axisymmetric beams with each an offset of $\pm 5.2 \mathrm{~cm}$ (in $\mathrm{X}$ and $\mathrm{Y}$ ) so that they formed a regular centered array. As expected due to a larger initial transverse kinetic energy for the beam ions, the minimum radius has been reduced compared to the $5.2 \mathrm{~cm}$ unique axisymmetric beam. This shows that focusing beam arrays (envisioned for other reasons) may eventually help to reduce the spot size. Another way of reducing it is to provide more neutralizing electrons by preionizing the background gas. Still on Fig.3, it is shown that preionizing $0.1 \%$ of the Flibe gas (case labelled $0.1 \%$ ) helps to focus a 5.2.cm axisymmetric beam on a $3 \mathrm{~mm}$ spot.

\section{Conclusion}

We have developed several tools allowing the modeling of final transport in the chamber with four level of description: a single envelope model (BENV), a multi-envelope model (BMENV), a slice PIC model (BPIC in R and XY modes) and a full PIC description (BPIC in RZ and XYZ modes). Using these tools, we have shown that the emittance has to be conserved has much as possible in the chamber and that under some reasonable conditions, it may not. We have also performed a parametric study of the minimum radius versus the gas ionization and beam stripping cross-sections which indicate, coupled with the analysis of 
envelopes snapshots and chosen quantities histories, that emittance growth and space-charge neutralization reduction to electron heating play a key role in pure ballistic transport through Flibe. Finally, it was shown that focusing a beam array or preionizing the Flibe gas may help in the reduction of the focal spot.

\section{References}

[1] M. Reiser, "Theory and Design of Charged Particles Beams", 1994, John Wiley and Sons; Inc.

[2] J.-L. Vay and C. Deutsch, A three-dimensional electromagnetic particle-incell code to simulate heavy ion beam propagation in the reaction chamber, Fus. Eng. Des., 32-33, 467 (1996).

[3] S. Mabong, Ph.D. Thesis, University Paris-XI, Orsay, France, (1996).

[4] J.-L. Vay and C. Deutsch, Charged compensated ion beam propagation in a reactor sized chamber, Phys. of Plasma, 5, 1190 (1998). 
a)

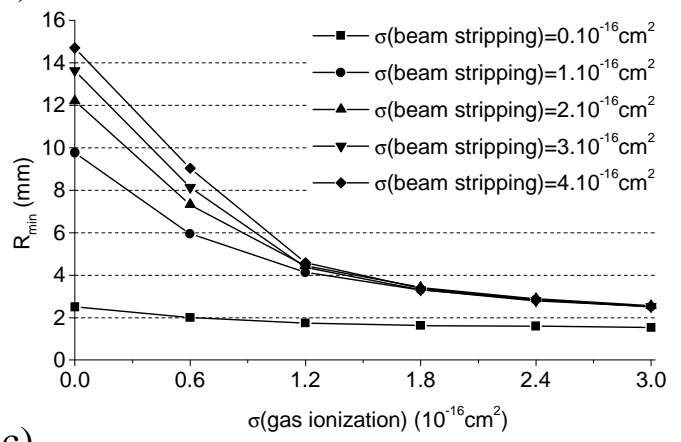

c)

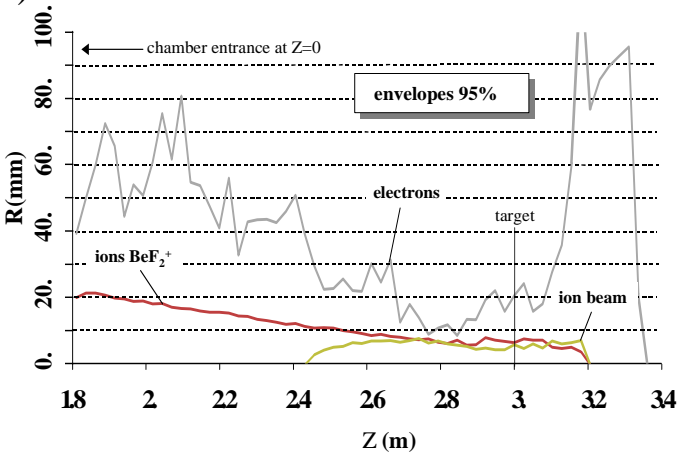

b)

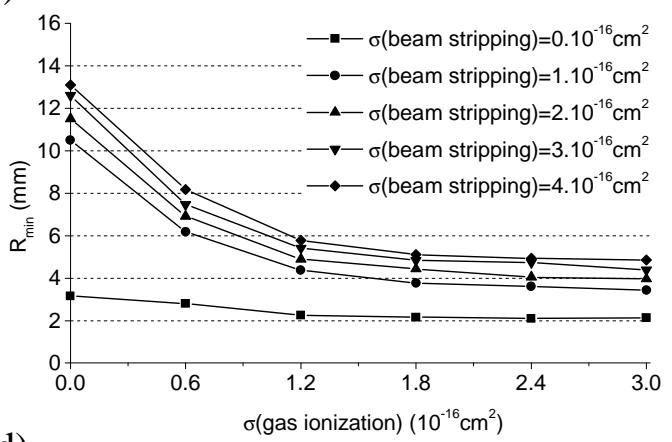

d)

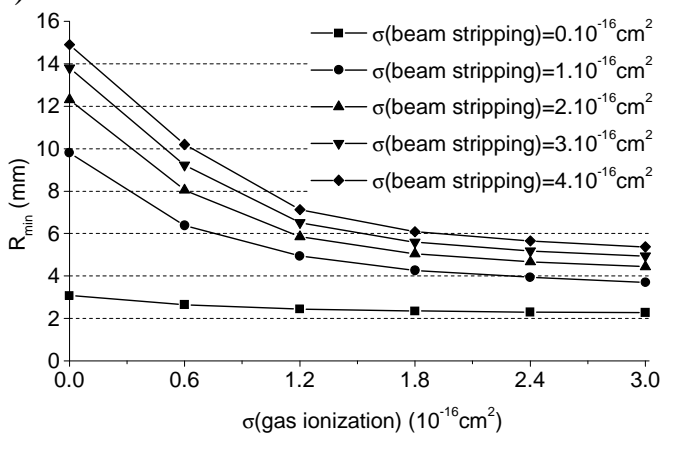

Figure 1: a) BMENV: min. radius versus cross-sections $(\varepsilon=$ const, $\mathrm{R}_{\mathrm{e} \min }=0$.), b) BPIC-RZ: min. radius versus cross-sections, c) BPIC-RZ: $95 \%$ envelopes snapshot, d) BMENV: min. radius versus cross-sections ( $\varepsilon=\mathrm{ramp}$, $\left.\mathrm{R}_{\mathrm{e} \min }=1 . \mathrm{cm}\right)$ 

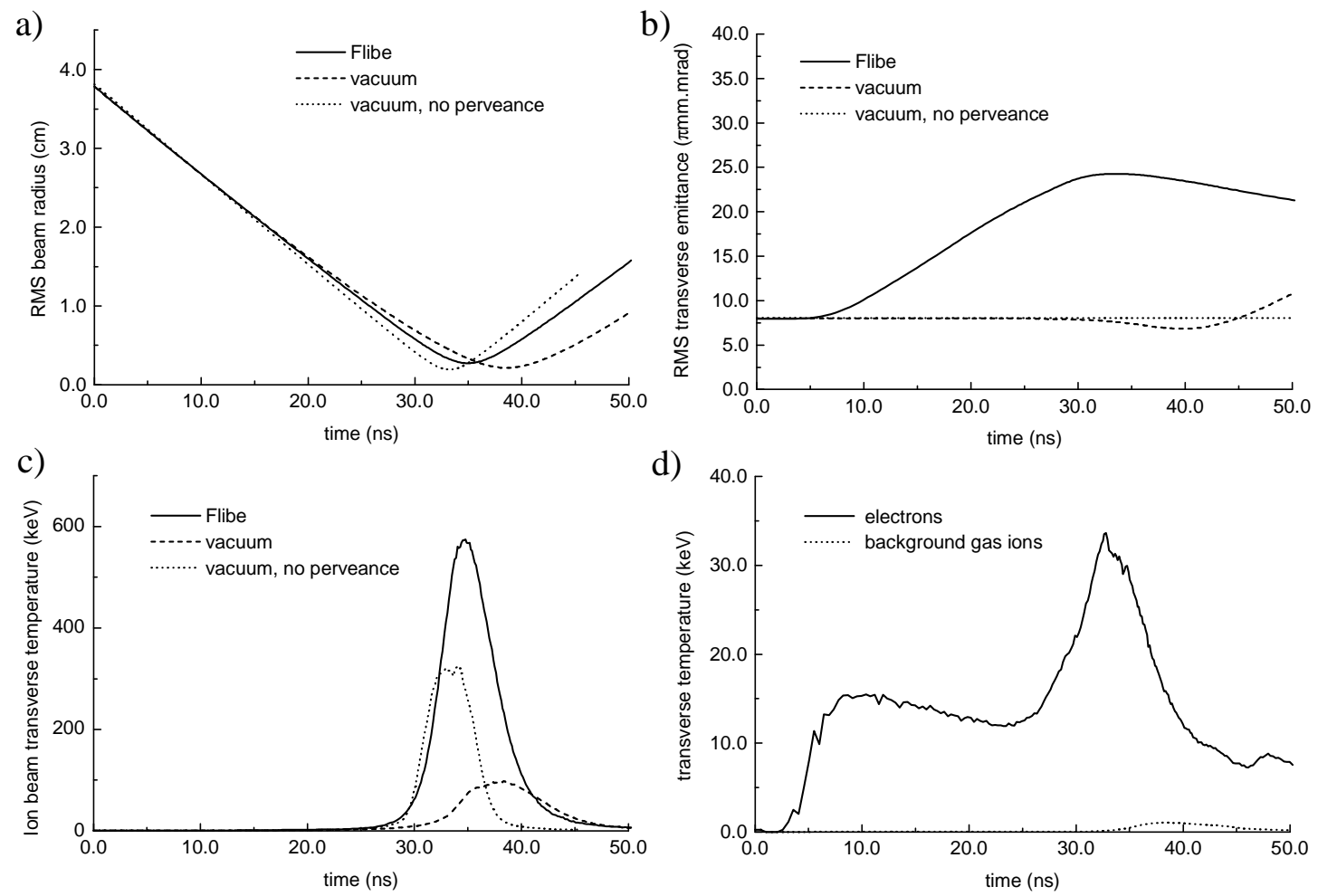

Figure 2: a) beam RMS radius history (BPIC-RZ), b) beam RMS emittance history (BPIC-RZ), c) ion beam temperature history (BPIC-RZ), d) electron temperature history (BPIC-RZ). Some of these results were also published in [4]. 


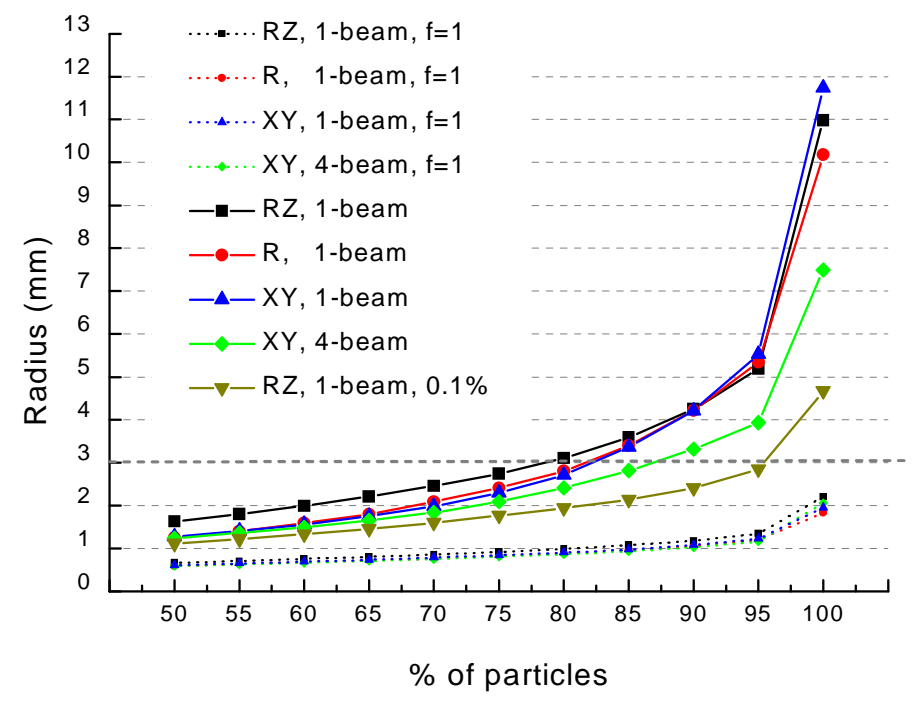

Figure 3: Minimum focusing radius as a function of a given number of inner particles. Four calculations are displayed without perveance $(f=1)$ and with perveance in different geometries (R, XY, RZ) either for a unique beam (1beam) or for an array of four beams (4-beam). 